\title{
ARGUMENTS
}

Cette rubrique veut offrir un lieu de discussion et de confrontation.

"Arguments» souhaite contribuer à un dialogue scientifique fécond en publiant des réactions à diverses publications scientifiques. Ces pages seront également ouvertes aux réflexions suscitées par les dossiers de la revue.

\section{A PROPOS DE "L'EMPIRISME IRRÉDUCTIBLE"}

\author{
Relecture de la postface d'Olivier SCHWARTZ (1993) \\ au livre de \\ Nels ANDERSON, Le Hobo. Sociologie du sans=abri1(1923)
}

Si on accepte l'idée déjà évoquée, entre autres par Margaret Mead, que "tout domaine soumis à l'apprentissage culturel ressortit à la communication" 2 , nous pouvons admettre qu'entre la communication (comprise comme approche scientifique) et l'anthropologie (ici l'ethnographie) se tissent des liens à la fois fondateurs et ultimes. C'est dans cette perspective que nous nous proposons de relire la postface qu'Olivier Schwartz a intitulée "L'empirisme irréductible". Nous souhaitons, à partir de ce texte et tout en connectant certaines des idées avancées par l'auteur aux travaux et réflexions d'autres ethnologues ou sociologues, offrir aux lecteurs quelques balises utiles et, bien sûr, discutables. A l'heure où, pour reprendre une expression d'Yves Winkin, "ces rustres [sociologues] d'outre-Atlantique qui ne

1 N. ANDERSON, Le hobo. Sociologie du sans-abri, Paris, Nathan, 1993.

2 Y. WINKIN, La nouvelle communication, Paris, Éd. du Seuil, 1981, p. 105. 
cessent de traîner leurs sabots sur de boueux «terrains»" 1 deviennent avec d'autres (les ethnologues travaillant sur le domaine européen, par exemple) les inspirateurs - parfois bien involontaires- de recherches nouvelles et empiriques dans le champ de la communication, il nous a paru intéressant de poser, à partir de la réflexion d'O. Schwartz, quelques repérages théoriques et épistémologiques. Si on considère que "la qualité ethnographique peut s'appliquer à tout type d'enquête qui repose sur une insertion personnelle et de longue durée du sociologue dans le groupe qu'il étudie" (p. 267), il n'est pas sans intérêt pour les chercheurs en communication de s'interroger de façon longue et approfondie sur les nécessités, les exigences et les limites de l'empirisme, compris comme paradigme et horizon de recherche. Notre relecture, à la fois sélective et ouverte, tente d'être un pas dans cette direction.

Notons, d'emblée, qu'O. Schwartz soutient l'idée que l'interactionnisme (Hughes, Goffman, Strauss...) -"en autorisant, grâce à un langage conceptuel très analytique, la décomposition des phénomènes sociaux en unités empiriques isolables- crée les conditions d'une très large observation des processus" (p. 292).

Il traite [les faits sociaux] non pas comme des «choses», mais comme des constructions liées à la manière dont les acteurs, placés dans des situations données, se définissent les uns par rapports aux autres, et élaborent pour ce faire le sens social des situations (p. 288).

Nous verrons, au cours de cet article, que l'auteur défend une idée d'élargissement de ce cadre. En "retournant" à Mauss, il pose que le cadre interactionniste n'est cependant pas le seul envisageable (ou envisageable seul) pour l'ethnographie telle qu'il l'appréhende et la conçoit.

Après une rapide présentation du livre de Nels Anderson, nous proposons d'instruire la réflexion autour de cinq concepts ou axes que nous considérons comme majeur, dans le cadre de cette lecture réappropriée: empirisme, situation, interprétation, réflexivité et transition.

1 lbid., p.104. 


\section{Présentation du hobo}

Septante ans après sa publication en langue anglaise, Le hobo. Sociologie du sans-abri, un des livres-pionniers de la sociologie qualitative américaine, a été récemment traduit et publié en français. Cette célèbre monographie des hobos (travailleurs migrants des États-Unis de la fin du $\mathrm{XIX}^{\mathrm{e}}$ siècle et du début du $\mathrm{XX}^{\mathrm{e}}$ ) participa à construire la réputation de l'école sociologique de Chicago qui, dans les années vingt, privilégiait la confrontation directe du sociologue avec les acteurs et les groupes sociaux qu'il étudiait. Reprenant les objectifs et les méthodes de l'approche ethnographique, les chercheurs de Chicago appliquèrent celle-ci non plus à l'étude des communautés lointaines et étrangères, mais à l'analyse systématique des modes de vie et d'organisation sociale qui avaient émergé, aux États-Unis, de la nouvelle organisation industrielle. "Les Anderson (1923), Cressey (1932), Lindeman (1924), Lynd (1922), etc. s'attachèrent, tour à tour, à s'intégrer dans des milieux urbains divers (petites et grandes villes, milieu des hobos, salles de danse, etc.), à y observer le déroulement de la vie sociale et à en chercher la signification à travers leurs échanges avec les acteurs sociaux concernés, leur participation à la vie de la communauté et une recherche documentaire fouillée, donnant lieu ainsi à des enquêtes complexes, d'une durée de quelques mois à quelques années. Leurs rapports d'enquête donnèrent de la réalité sociale une image dense visant à refléter, à travers une analyse minutieuse des situations et des événements sociaux observés, la complexité du réel et des enjeux sociaux, et la traduction de ces enjeux dans la vie des membres des communautés étudiées. L'observation directe tendait donc, à cette époque, à se présenter comme une méthodologie «complète» d'approche du réel, voulant allier à l'appréhension intersubjective des situations sociales étudiées une analyse objective de leur dynamique, basée sur la confrontation systématique de données de sources diverses"

En suivant le texte de Nels Anderson, ancien hobo lui-même, le lecteur se trouve projeté au cœur de la vie de ces "hommes qui partent de chez eux" pour se retrouver tantôt sur les routes, tantôt, comme en attente, dans certains quartiers de Chicago. Vieux de septante ans, le texte n'a rien perdu de son intérêt documentaire. Les questions

1 B. GAUTHIER (dir.), Recherche sociale. De la problématique à la collecte de données, Montréal, Presses de l'Université du Québec, 1987, p. 227. 
méthodologiques qu'il ne manque pas de soulever sont reprises et explorées par Olivier Schwartz dans la longue et très intéressante postface intitulée "L'empirisme irréductible" que nous allons explorer dans cet article.

Avant de nous arrêter à cette postface, resituons brièvement le travail de Nels Anderson qui étudia, dans le cadre d'une recherche effectuée suite à une demande des institutions sociales, la "Hobohème" comme quartier de repos, de passage ou de repli pour ces ouvriers nomades. Bien qu' "ethnologue indigène" plus exactement indigène ethnologue, l'auteur s'efforce, loin d'une perspective misérabiliste, de saisir ce groupe social dans toutes les dimensions de sa vie concrète: travail, logement, nourriture, finances, santé, rêves et plaisirs...

Par la richesse de ses descriptions, par la profondeur de son regard, le livre d'Anderson met aussi en évidence l'irremplaçable apport qui peut être celui de l'«ethnologie indigène» aux sciences sociales. Il est, certes, parfaitement clair que la sociologie ne peut se passer de "professionnels", conduisant leurs enquêtes dans le cadre d'un questionnement d'ensemble, rapportant leur matériaux à des catégories abstraites, combinant proximité et distance à l'objet. Mais ceux qui se sont essayés à l'enquête sur le «terrain» savent bien que celui-ci est d'accès difficile, que les matériaux significatifs y sont rares, et que le sociologue éprouve souvent de la peine à rassembler les conditions pour se faire ethnologue. Pourquoi dès lors ne pas prendre appui sur le savoir de l'ethnologue indigène, pourquoi ne pas permettre à celui-ci de se faire, dans une certaine mesure au moins, sociologue? On objectera sans doute, et avec raison, qu'un savoir né dans ces conditions a toutes les chances d'être chargé d'affectivité inconsciente, et qu'il faudrait donc aussi le mettre en question. Certes. Mais cette objection pertinente ne doit pas masquer que d'irremplaçables réserves d'intelligence sociologique existent en dehors de la sociologie professionnelle, notamment du côté de ceux qui, membres indigènes d'un milieu donné, doivent à la singularité d'une position ou d'une histoire la capacité de regarder avec

1 "La situation dans laquelle se trouve Anderson au moment de son travail de «terrain» n'est pas celle d'un ethnologue professionnel qui s'efforce de pénétrer de l'extérieur un groupe auquel il est étranger. L'enquête d'Anderson repose sur un tout autre cas de figure. Elle résulte d'une série de contingences au terme desquelles un individu singulier se fait l'ethnologue de son propre milieu, celui auquel il appartient encore largement même s'il est engagé dans la tentative incertaine et difficile d'en sortir" ("Présentation" d'O. SCHWARTZ, p. 13). 
pénétration leur univers et de communiquer ce qu'ils ont su voir. Jamais la sociologie ne parviendra à se passer d'un tel savoir $(\ldots)^{1}$.

Ajoutons qu'il nous paraît que le texte de Nels Anderson révèle trois caractéristiques essentielles et constitutives de son propos et de son positionnement. D'abord, l'usage de la description à la fois comme principe de mise en évidence et de suggestion intreprétative. Prenons un exemple. Parlant de l'attrait qu'exercent sur les "jeunes hommes sans attache" les charmes du travail saisonnier qui enflamme l'imagination, il recourt à la présentation d'un cas particulier.

Cinquante-huit ans, né en Belgique. Il est arrivé dans ce pays avec ses parents en 1882. Sa famille s'est installée dans une ferme au nord du Wisconsin, où ils allaient habiter pendant plusieurs années. Gamin, il passait son temps libre à travailler dans les bois. Son père s'est bientôt lassé de la culture et, comme il avait été mineur en Belgique, il a décidé que sa place était plutôt dans les mines de charbon du sud de l'Illinois. Une fois la famille installée, le jeune garçon s'est trouvé incapable de tenir en place dans cette ville minière, et a décidé de retourner dans son ancien pays du Wisconsin, afin de trouver un travail dans les bois, plus conforme à ses goûts. Pendant plusieurs années, il a partagé son temps entre les bois du nord en hiver, et, en été, les mines de l'Illinois où résidait sa famille. Cependant, il ne s'est jamais fait au travail dans les mines de charbon, et au bout d'un certain temps, il s'est mis à faire la moisson en guise de travail d'été. Parfois, il travaillait sur des chantiers de construction ferroviaire ou à diverses activités saisonnières. Il a passé plusieurs hivers à Chicago et généralement (selon ses dires), il est parvenu à s'en sortir financièrement. Cette année toutefois (1921-22), il lui est arrivé de prendre ses repas aux missions.

Ce cas particulier met en évidence les étapes qui font passer de l'état de travailleur saisonnier stable à celui de travailleur migrant. Il suggère comme il est simple et naturel pour un travailleur migrant de tomber plus bas encore sur l'échelle économique et de finir chaque hiver en Hobohème à «parasiter les missions»" (p. 92).

Les deux autres caractéristiques sont le plus souvent relativement entremêlées. En effet, l'explication multiple et contextuelle qui met en action des cadres interprétatifs globaux (socio-économiques, par

1 Ibid., p. 19-20. 
exemple) se trouve assez souvent développée en regard d'une perspective d'action (intervention) sociale -qui correspond, rappelons-le, à la perspective de la demande de recherche-. L'extrait qui suit nous paraît bien illustrer ce point de vue.

Ce processus de dégradation personnelle du travailleur occasionnel migrant, qui le conduit de l'indépendance économique au paupérisme, n'est qu'un aspect du rôle joué par les forces économiques dans la société industrielle moderne. Les industries saisonnières, les cycles économiques, l'alternance des périodes d'emploi et de chômage, la précarisation de l'industrie ont donné naissance à cette grande armée de réserve industrielle constituée d'hommes sans logis et sans attache qui se concentre en période de sous-emploi -comme en hiverdans les centres stratégiques de transport, c'est-à-dire dans les plus grandes de nos villes. Il faut bien que ces hommes vivent; la majorité d'entre eux sont indispensables dans l'organisation actuelle de l'industrie fondée sur la compétition; des associations et des personnes, mues par un élan religieux et philanthropique, continueront à tenter d'adoucir leur condition; et pourtant, leur concentration en nombre croissant, l'hiver, dans certains quartiers de nos grandes villes, ne peut nous apparaître que comme une menace. Toutefois, la politique consistant à laisser «se débrouiller» les travailleurs occasionnels migrants reste pour le moment plus facile et moins coûteuse, même si l'adoption de mesures préventives contre la dégradation économique et morale des sans-domicile renforcerait, à longue échéance, l'équilibre social et l'économie nationale (p. 88).

Après cette très rapide évocation de la recherche et du livre de Nels Anderson, passons à notre relecture-réarticulation de la postface d'Olivier Schwartz.

\section{Empirisme}

Traiter des ambitions épistémologiques de la recherche ethnographique qui semble parvenue aujourd'hui à un certain degré de réflexion critico-méthodologique: voilà, en quelques mots, les objectifs de cette postface. Autrement dit, elle pose, comme en négatif, la question suivante: "Faut-il considérer que la pratique ethnographique est aujourd'hui en mesure de se libérer de cet empirisme tâtonnant qui semble être sa maladie infantile, et que l'heure a sonné pour elle d'entrer dans l'âge de la «rigueur», en donnant à ce terme le sens d'un 
«modèle fort», qui n'accepterait pour légitimité que des opérations scientifiquement bien fondées?" (p. 265). On le voit d'emblée, la définition de l'empirisme est au cœur de la réflexion. Olivier Schwartz ne l'envisage pas comme un positivisme tranquille qui croirait pouvoir s'arrêter strictement aux faits et il le définit de façon à la fois précise et ouverte.

Le terme d' «empirisme» peut d'abord évoquer, pour le lecteur, cette attitude naïve qui consiste à croire que les données immédiates des sens constituent la base ferme de toute connaissance. Idée évidemment critiquable.

Mais l'empirisme renvoie aussi à une tradition philosophique construite et argumentée, qui a ses lettres de noblesse et qui n'est nullement naïve. Un intérêt majeur de celle-ci est de développer ce que l'on pourrait appeler une image «non sublime» du savoir. Derrière ce qui se présente sous le nom de «raison», de «vérité nécessaire» et de «science», l'empirisme met en évidence l'action de principes profanes, plus limités et sans doute plus faillibles que ne l'accorderaient des philosophes d'orientation «rationalistes». On peut songer ici à Hume et à sa célèbre analyse du rôle de l'habitude dans nos jugements de causalité. Cela ne signifie nullement que le savoir soit renvoyé au côté de l'incertitude: simplement, son existence n'autorise pas à le considérer comme un domaine absolument spécial de l'activiré de l'esprit, ni à surestimer son extension ou la valeur de ses fondements.

L'empirisme peut conduire au scepticisme (...). Nous allons plutôt le développer dans le sens d'une épistémologie «tolérante»: renonçant à un modèle excessivement fort de rigueur, et acceptant l'idée qu'une démarche de connaissance peut être conduite à incorporer bien des éléments et des opérations qui relèvent, certes d'une forme de jugement réfléchi, mais qui ne peuvent pas se voir attribuer la nécessité de ce qui est scientifiquement fondé (p. 266).

Le positionnement général d'O. Schwartz se trouve ainsi exprimé au travers de cette proposition définitionnelle. Sans entrer ici dans une discussion sur le "scientifiquement fondé" ou, mieux, le scientifiquement fondable, il nous paraît que, d'une certaine façon, la position définie par l'auteur appelle, comme en écho et en prolongement, ce que Norbert Elias appelait "l'incertitude [qui] règne parmi les sociologues à propos de l'objectif de la recherche scientifique". "Selon moi, écrit-il, cet objectif est commun à toutes les sciences: faire connaître aux êtres humains quelque chose qu'ils ignoraient, 
faire progresser le savoir humain, le rendre plus exact ou mieux adapté; en termes un peu plus techniques, il s'agit d'étendre l'ensemble des symboles humains à des domaines non encore étudiés. Or l'objectif des sciences -la découverte- a été fortement obscurci par des discussions formalistes sur la «méthode» de la recherche scientifique. Ce déplacement de la discussion -de l'objectif et de la fonction de la recherche scientifique à la méthode- est, en termes sociologiques, symptomatique d'une lutte de pouvoir. Des spécialistes des sciences de la nature, ainsi que des philosophes des sciences convaincus de la primauté des sciences de la nature, ont usé de leur pouvoir, aussi bien intellectuel que social, pour persuader les autres que la «méthode» employée dans les sciences naturelles, en particulier en physique classique, est la seule légitime pour aboutir à une découverte scientifique. Les défenseurs de cette position, qui n'ont souvent qu'une maigre expérience de la recherche en sciences sociales, s'inspirent de principes philosophiques ou d'une tradition de l'«histoire des idées». Il faut donc préciser sans équivoque que l'on peut faire avancer la connaissance et réaliser des découvertes dans le champ de la sociologie par des méthodes très différentes de celles que l'on utilise dans les sciences de la nature. C'est la découverte, et non la méthode, qui légitime la recherche comme science"1.

D'autre part, si on suit G. Gurwith et son "empirisme radical", "c'est-à-dire le complet respect de la multiplicité des expériences que la vie collective ne cesse d'engendrer et de modifier"2, on prend alors «empirique» "au sens fort du terme [comme] seul moyen de rendre compte de la complexité des situations de communication"3. Il n'y a, dès lors, évidemment pas d'ethnographie de la communication possible sans recours à l'expérience, sans plongée à la rencontre des acteurs, sans ce que Paul Rabinow appelle "l'alchimie corrosive du terrain" 4 . Cela pose, de manière fondamentale, la question de la place et du statut du chercheur-observateur. Nous y reviendrons. Mais il nous faut, avant cela, prendre au sérieux la mise en garde d'Alain Ehrenberg: "Dans tout phénomène humain, écrit-il, nous pouvons lire des représentations qu'une société se fait d'elle-même. Des

1 N. Elias, "Introduction", Sport et civilisation (N. Elias, E. Dunning), Paris, Fayard, 1994 (1ère éd. 1986), p. 26-27.

2 P. ANSART, Les sociologies contemporaines, Paris, Éd. du Seuil, 1990, p. 12.

3 B. MièGE (sous la dir. de), "Plaidoyer pour des problématiques transversales et partielles", Médias et communication en Europe, Grenoble, PUG, 1990, p. 19.

4 P. RABINow, Un ethnologue au Maroc, Paris, Hachette, 1988, p. 17. 
représentations et non la représentation, le problème étant alors de les identifier. Le dogmatisme interprétatif guette sans doute le chercheur qui veut voir du global dans le local, mais pas plus que celui qui croit se cantonner au terrain sûr de l'empirisme minutieux, oubliant que les faits contiennent toujours de l'interprétation. L'illusion spéculative et l'illusion empiriste sont constitutives des sciences humaines et aucune méthode ne nous garantit absolument contre ces dangers"1.

\section{Situation}

Ce qui est dit à l'ethnographe, “ce qui lui est donné à voir n'est jamais dissociable des caractéristiques spécifiques de la situation d'enquête" (p. 272). L'ethnographie, pour Olivier Schwartz, "s'applique de manière intensive à l'étude de groupes et d'individus saisis dans des cadres localisés. Les propriétés des faits qu'elle observe méritent, à cet égard, d'être qualifiés de «situationnelles»" (p. 299).

Toute approche empirique de la communication est, dès lors, situationnelle dans la mesure où elle se préoccupe d'étudier des phénomènes divers mais toujours en situation. On peut entendre par ce dernier terme, dans un sens très proche de la définition goffmanienne, toute zone matérielle, spatiale et temporelle où des actants se trouvent mutuellement en possibilité d'interagir ${ }^{2}$.

En fait, la situation est "engendrée par ces activités concomitantes" 3 et les acteurs "concourent à lui donner un sens cohérent et transmissible"4. Parler de situationnalité, c'est aussi marquer, d'une certaine façon, "l'indexicalité5 de toute situation constituée en objet d'analyse"6 et, dans le même temps et pour ne pas tomber dans un localisme radical, mener une approche contextualisante. En d'autres mots, réunir dans l'analyse le ponctuel et l'englobant.

1 A. EhrenBerg, Le culte de la performance, Paris, Calmann-Lévy, 1991, p. 34.

2 E. Goffman, Façons de parler, Paris, Éd. de Minuit, 1987, p. 91.

3 C. JAVEAU, La société au jour le jour, Bruxelles, De Boeck, p. 73.

4 Ibid.

5 En suivant les ethnométhodologues, on peut entendre par "indexicalité", la "frange d'incomplétude" propre à toute situation qui implique la nécessaire prise en considération de la dimension contextuelle (ainsi que du cadre) afin d'accéder au sens.

6 G. LAPASSADE, L'ethnosociologie, Paris, Méridiens Kliencksieck, 1991, p. 81. 
En suivant I. Joseph ${ }^{1}$, nous pouvons dire que les effets de situation sont d'ordre conjoncturel et que les effets de contexte sont d'ordre structurel. Cette perspective situationnelle marque le primat de l'acteur, du présent et de l'émergence. Cependant, loin d'ignorer les "formes englobantes", il faut tout de suite insister, même si toute situation est unique et particulière, sur le poids du contexte (et du cadre) dans toute situation et sur ce que cet auteur appelle, reprenant le terme à Erving Goffman, des "inerties situationnelles" qui sont des formes répétitives, bricolées sur base de situations déjà vécues, en quelque sorte déterminées par le contexte et par le cadre. Toute recherche ethnographique dans le champ de la communication devra, nécessairement, se pencher avec le plus grand soin sur ces formes d'inerties.

Dans le même ordre d'idées, $O$. Schwartz défend le point de vue que les faits ethnographiques "s'inscrivent dans des rapports d'ensemble, dans les modes de fonctionnement caractéristiques d'une société globale. Ils présentent nécessairement des propriétés qui expriment leur appartenance à l'ordre social, et qui relèvent de leur enchâssement dans ses mécanismes" (p. 299). Cherchant la profondeur et la transversalité des faits ethnographiques, l'auteur propose ce qu'il appelle une "ligne maussienne comme voie vers une sociologie compréhensive". Il qualifie, en reprenant les termes de Paul Veyne, cette voie de "rétrodictive", c'est-à-dire s'efforçant, "à partir d'un ensemble diversifié de matériaux considérés comme des effets, de régresser vers des «raisons sous-jacentes» et non données empiriquement". Cette ambition de lire les matériaux ethnographiques dans une perspective plus structurelle fait très explicitement référence à la vision de Marcel Mauss² ("une science sociale de l'observé"), au fait social total, mais aussi aux travaux plus récents de Gregory Bateson ou de Pierre Bourdieu. Cette dimension inférentielle et inductive, si elle doit sans cesse être mise en confrontation avec le donné empirique, n'en reste pas moins toujours soumise à une forme de "bricolage savant" qui doit accepter de se soumettre, en complément indispensable à la perspective rétrodictive, à un nécessaire retour aux

1 I. JOSEPH, "L'analyse de situation dans le courant interactionniste", Ethnologie française, vol.XII, n², 1982, p. 229-234.

2 Olivier Schwartz fait une autre référence explicite à Marcel Mauss quand il insiste sur le fait que "la transversalité du fait ethnographique (...) signifie qu'il faut se garder d'imposer aux activités un découpage qui viole les cadres, ou les ensembles à l'intérieur desquels elles sont effectivement vécues par les individus"' (p. 296). 
situations qui seules permettent de déceler que les acteurs, loin d'être uniquement soumis aux pesanteurs structurelles "sont aussi des acteurs de leur vie" (p. 302).

\section{Interprétation}

Une des questions centrales qui "travaille " (de l'intérieur comme de l'extérieur) la démarche ethnographique repose sur la production et la validité de la construction des données à partir desquelles l'interprétation va s'élaborer. En rappelant que le "premier objet de l'enquête n'est pas de répondre à des questions mais de découvrir celles qu'on va se poser" (p. 281), O. Schwartz montre que, dans la pratique ethnographique concrète, "l'observation ne se fait pas nécessairement selon des hypothèses précises, et [que] les données sont pour partie des traces lacunaires, liées aux incertitudes de la mémoire" (p. 282). On le voit, cette position pose, de façon évidente, la question du statut épistémologique des données. En effet, en insistant sur l'importance de faire participer les "données vérifiées", les choses dites par les "observés" et les choses retenues -(re)construitespar l'observateur au même processus interprétatif, l'auteur propose, dans le même mouvement, d'admettre que les données "comportent plusieurs classes d'éléments affectés d'indices de certitude et de fonctions heuristiques différentes" (p. 284).

Il ne s'agit donc, en aucun cas, de faire "l'impasse sur tout ce qui constitue le «hors-champ» de ce qui est décrit, c'est-à-dire la mise en perspective des usages dans le temps et dans l'espace social (...). Cette mise en perspective permet de dégager le sens des usages, et d'en dire autre chose que ce qu'on en perçoit quand on reste aux apparences, ou de ce qu'on y projette quand on fait l'économie de l'observation minutieuse. En quelque sorte, l'analyse micro-sociale que nous proposons, c'est de se situer ni trop près, ni trop loin des usages concrets et des représentations des usagers, pour en faire émerger des significations pertinentes"

De cette manière, l'ethnographe n'est " ni celui qui voit «tout de l'intérieur», ni celui que tout le monde regarde. Il est régulièrement renvoyé aux limites de son savoir et de sa participation. L'oublier, c'est céder à la mystique du «terrain», qui sans doute dit quelque

1 J.-C. Baboulin, J.-P. Gaudin, P. Mallein, Le magnétoscope au quotidien, Paris, Aubier Montaigne, 1983, p. 32. 
chose de ce que l'on y cherche, mais qui rend bien mal compte de ce que l'on y trouve" (p. 271).

Olivier Schwartz, précisant que l'ethnographie est une pratique irréductiblement interprétative et que "la machine à interpréter fonctionne facilement à l'état «sauvage»” (p. 285), invite à la prudence en proposant de baliser cette opération de contraintes qui l'obligent à différer l'induction. La notion de "saturation", développée par des auteurs comme Anselm Strauss ou Daniel Bertaux, met clairement en évidence cette obligation.

Lorsqu'une hypothèse, par rectifications successives, atteint un degré de pertinence, vient un moment où les données empirique diverses lui apportent une confirmation régulière. Cette régularité, est-il besoin de le dire, n'a jamais rien d'une «loi» de la nature, mais renvoie simplement à la notion approximative de «bonne résistance» dans des situations empiriques variées. Alors on peut estimer que cette hypothèse est «saturée», c'est-à-dire qu'elle se comporte suffisamment bien au cours de nombreuses mises à l'épreuve pour pouvoir être considérée comme fiable (p. 286).

\section{Réflexivité}

Les ethnologues, et avec eux la plupart des chercheurs en sciences sociales ${ }^{1}$, savent depuis bien longtemps que l'observateur fait partie de son observation et que la prise en compte de l'observateur (de son rôle, de son action...) dans le processus même de la recherche, même si elle n'est véritablement pas neuve, n'en reste pas moins vitale. "Déjà chez Michel Leiris, dans L'Afrique fantôme $e^{2}$, on trouve l'idée que la seule façon d'être honnête dans son regard anthropologique est de s'analyser soi-même. Il s'agit de l'observateur, en tant qu'il est représentatif de quelque chose"3. Ce même Michel Leiris faisait d'ailleurs de "l'opacité des relations avec les membres des

1 Olivier Schwartz reprend, à ce propos la très parlante question de W. LABOV (Le parler ordinaire, Paris, Éd. de Minuit, 1978): “Comment observer la façon dont les gens parlent quand on ne les observe pas?"

2 M. LEIRIS, L'Afrique fantôme, Paris, Gallimard, 1934.

3 M. AuGÉ, entretien accordé à Edwin Zaccai, "Les grands voyages proches de Marc Augé", Intermédiaire, n³6, 1992, p. 8. 
groupes étudiés [et] de sa difficulté à appréhender le sens de ce qu'il observait, une composante essentielle de sa démarche"l.

"L'observateur est toujours indexicalement inclus", toujours situationnellement impliqué et en cela "responsable de l'image de la collectivité qu'il diffuse, comme il l'est de la visibilité à laquelle il la contraint de fait, par son métier même"'3. Conscient de cette responsabilité, il devra effectuer un nécessaire retour réflexif qui pourra peut-être éviter que "la pratique scientifique [ne] demeure hors champ"4. Cette perspective critique-analytique est, à la suite d'auteurs comme Gérard Althabe, Jeanne Favret-Saada, Paul Rabinow et bien d'autres, défendue par Olivier Schwartz. Insistant sur le fait que l'objet d'étude n'a aucune espèce de transparence et que le travail d'enquête ethnographique ne garantit en rien une forme de dévoilement automatique, il écrit à propos de la démarche critiqueanalytique:

Elle se caractérise d'abord par un principe de lecture et de décryptage des données d'ethnographie, qui consiste à placer la situation d'enquête et ses effets au centre de l'analyse des matériaux. Puisqu'il n'y a pas d'observation neutre et que les «données» ne sont jamais dissociables des dynamiques à l'œuvre dans la recherche elle-même, il n'y a donc pas d'autre voie pour les comprendre que de suivre réflexivement ce qui se joue dans la relation du sociologue à ses enquêtés, d'identifier les rôles qu'on lui attribue, et de rapporter ce qu'on lui dit ou ce qu'on lui montre à la place momentanée qu'il occupe dans le jeu d'interactions suscitées par sa présence. Les matériaux d'enquête doivent donc d'abord être traités comme des effets de la situation d'enquête, et non comme des représentations immédiates d'une réalité «naturelle», antérieure à l'observation. En adoptant cette attitude méthodologique, on se donne les moyens d'une lecture «non naïve» des phénomènes observés ou des propos recueillis, capable d'opérer à la fois la démystification et le décryptage des «données» en fonction des conditions dans lesquelles elles ont été produites (p. 275).

1 M. Clavel, "L'ethnologie urbaine en France: des sociétés exotiques à l'espace urbain contemporain", L'homme et la société, n¹04, 1992, p. 107.

2 J. WIDMER, Langage et action sociale, Fribourg, Presses universitaires de Fribourg, 1986, p. 39.

3 M. Clavel, op. cit., p. 101.

4 B. Latour, Nous n'avons jamais été modernes, Paris, La Découverte, 1991, p. 141. 
Selon ce point de vue, l'ethnographe doit alors nécessairement allier "conscience critico-méthodologique de ses démarches et tolérance à l'impureté de ses matériaux, donc à la contingence des résultats" (p. 271). Mais O. Schwartz va cependant au-delà de ce strict constat quand il préconise de ne pas envisager ce "paradoxe de l'observateur" de façon tyrannique ou maximaliste. En insistant sur la nécessité du questionnement critique, il propose néanmoins d'en atténuer la rigidité (qui pourrait devenir sclérosante) en mettant en avant la capacité qu'à le chercheur de moduler (de jouer sur) les effets induits par l'observation et la nécessité de ne pas faire de tout matériau d'enquête le seul produit de la situation d'enquête. Pour les "observés", en effet, les contraintes et les habitudes quotidiennes ne s'effacent pas (complètement) sous l'action des contraintes -non ordinaires- de la situation d'enquête puisqu'ils ont le plus souvent intérêt à maintenir (du moins en partie et, en tout cas, sur la durée ${ }^{1}$ ) les exigences, les engagements, les cadres qui sont ceux de leur vie ordinaire, c'est-à-dire de leur vie sans ethnographe qui les observe. Ainsi, "quelles que soient les données, on s'interrogera toujours sur ce qu'elles peuvent devoir à la situation d'observation, mais on ne s'interdira pas d'y chercher les indices de réalités indépendantes de l'enquête"' (p. 279).

\section{Transition}

Sortant quelque peu de la stricte relecture du texte d'Olivier Schwartz, notons qu'Alfred Schutz défend une conception sensiblement différente de celle de Nels Anderson qui, rappelons-le, était hobo bien avant d'être ethnologue. Pour lui le détachement de l'observateur signifie "qu'il n'est pas impliqué dans les espoirs ni dans les craintes des acteurs qu'ils se comprennent ou non, qu'ils arrivent ou non à leurs fins (...). Ainsi son système de pertinence diffère de celui des gens qui sont in media res et l'habilite ainsi à voir plus et moins qu'eux"2 mais toujours à partir de ce qu'ils voient ou disent voir. La distanciation ou le détachement permet alors au chercheur qui

1 "La durée définit précisément l'espace de l'enquête (...). Le temps et la quotidienneté sont deux agents puissants de banalisation de l'ethnographe (...). Le caractère perturbateur de l'observation, même s'il est peu vraisemblable qu'il disparaisse jamais, peut se réduire" (p. 278).

2 A. SchuTz, Le chercheur et le quotidien, Paris, Méridiens Klincksieck, 1987 (1ère éd. 1971), p. 33 et sv. 
est pris par sa recherche et les sujets de se déprendre. Mais se déprendre, ce n'est pas rompre. Loin de là. C'est se donner la possibilité d'être repris en conscience et ainsi de suite. Il en va du processus d'interprétation comme de la présence au terrain: y plonger n'est pas s'y noyer et se déprendre, n'est pas rompre. Expliquons-nous.

Jean-François Lyotard, présentant la phénoménologie et traitant des risques et des limites de la compréhension immédiate, souligne que les "types de compréhension «évidente» et spontanée résultent en réalité des sédimentations complexes de notre histoire individuelle et de l'histoire de notre culture"'. Toute compréhension immédiate étant située et marquée (ce qui ne la rend pas inintéressante ni inutile), ce que nous appellerons la transition épistémologique (axée sur la prise de distance sans rupture) devient l'opération par laquelle une compréhension de second degré (qui s'exerce sur ce qui a été dégagé et décrit de la compréhension des acteurs par un processus définissable et contrôlé) peut advenir.

Nous employons le terme transition épistémologique en regard de celui, classique, de "rupture épistémologique". Se référant à Bachelard ${ }^{2}$, Louis Moreau de Bellaing, dans sa critique de l'empirisme en sociologie, écrit que "la rupture épistémologique consiste à rompre avec le donné, avec le matériau accumulé et traité, voire déjà interprété en interprétation dérivée [c'est-à-dire sans théorie] pour pouvoir le penser" 3 . Pour nous, du "sens commun" ou du sens donné par les informateurs au sens donné par l'ethnographe au sens commun, il n'y a pas nécessité de brisure, d'éloignement irrévocable mais plutôt nécessaire continuité et passage, d'où le terme de transition. Ce terme se renforce encore par l'affirmation de l'état intermédiaire -c'est-à-dire dynamique et en voie de continuelle transformation- et non fini des propositions compréhensives en regard de la recherche de la vérité démontrée (ou falsifiable) poursuivie par les tenants de la rupture.

Dans cette perspective, toute tentative théorique (du particulier au global) doit être conçue comme inachevée et a-dogmatique. Et, ici,

1 J.-F. LyOTARD, La phénoménologie, Paris, P.U.F., 1986, p. 76.

2 Pour une présentation synthétique du concept de "coupure (ou rupture) épistémologique", révélatrice de "la démarcation par laquelle la science se sépare de l'idéologie", on pourra se référer, entre autres, à J.-P. RoY, Bachelard ou le concept contre l'image, Montréal, Presses de l'Université de Montréal, 1977.

3 L. Moreau de Bellaing, L'empirisme en sociologie, Paris, L'Harmattan,1992, p. 20. 
nous pouvons partager l'avis de L. Moreau de Bellaing en ce qu'il met en garde contre des velléités hégémoniques et reconnât (du moins partiellement) les apports d'une approche qui diverge radicalement de la sienne: "Les empiristes en restent à ce que les philosophes appellent la doxa. Ils dérivent leurs interprétations des faits euxmêmes et de l'organisation des faits (...). Que les apports de la doxa soient déjà importants pour une recherche, bien des travaux de sociologie et de sciences sociales actuels peuvent en témoigner. Rien ne s'oppose à ce qu'un chercheur s'arrête à la doxa, mais à condition de ne pas prétendre que c'est cela et cela seulement la sociologie"'.

\section{Perspective}

Dans la perspective d'un déploiement scientifique et méthodologique non nécessairement astreint aux anciennes balises, tenter de pratiquer la démarche ethnographique dans le champ des études en communication, c'est, dans un même mouvement, s'efforcer de la mieux délimiter, laisser sa place à "l'inachevé et à l'incertitude"2 ainsi qu'à l'imagination sociologique telle que la conçoit Liliane Voyé: "C'est l'ouverture sur les questions non rhétoriques à partir de la mise en œuvre d'une perspicacité qui pourra être d'autant plus grande que sera large la connaissance de la réalité sociale dans son histoire et dans ses multiples aspects. C'est aussi le rejet du "prêt-à-porter» sociologique au bénéfice d'un bricolage sans cesse réinventé qui seul ouvrira à de nouvelles questions" 3 .

Dans une perspective, somme toute assez proche, O. Schwartz écrit que "la situation de l'ethnographe pourrait finalement se définir par une sorte d'ambivalence épistémologique. Il lui faut une «conscience», un capital de réflexions et d'exigences méthodologiques le contraignant à critiquer, à évaluer ses résultats; mais il lui faut aussi, pour en obtenir d'autres, un modèle suffisamment souple, qui tolère une part de «bricolage», de contingence, d'incertitude" (p. 267).

\section{Gérard DERÈZE}

Ibid., p. 56.

2 M. AUGE, Symbole, fonction, histoire. Les interrogations de l'anthropologie, Paris, Hachette, 1979, p. 207.

3 L. VOYE, "Méthodes et imagination sociologiques", Recherches sociologiques, vol. XVI, n² 2, 1985, p. 193. 